\title{
ERRATUM
}

\section{Transcription Factor Binding and Induced Transcription Alter Chromosomal c-myc Replicator Activity}

\author{
M. Ghosh, G. Liu, G. Randall, J. Bevington, and M. Leffak \\ Department of Biochemistry and Molecular Biology, Wright State University School of Medicine, Dayton, Ohio
}

Volume 24, no. 23, p. 10193-10207, 2004. Page 10203, column 1, line 5: "promoter results" should be deleted. 